\title{
Optical dipole-force cooling of anions in a Penning trap
}

\author{
Julian Fesel, ${ }^{*}$ Sebastian Gerber, and Michael Doser \\ CERN, European Laboratory for Particle Physics, 1211 Geneva, Switzerland
}

\author{
Daniel Comparat \\ Laboratoire Aimé Cotton, CNRS, Université Paris-Sud, ENS Paris Saclay, Université Paris-Saclay, Bâtiment 505, 91405 Orsay, France
}

(Received 21 December 2016; revised manuscript received 17 July 2017; published 13 September 2017)

\begin{abstract}
We discuss the possibility of using optical dipole forces for Sisyphus cooling of ions stored in a Penning trap by addressing the specific case of the molecular cooling candidate $\mathrm{C}_{2}{ }^{-}$. Using a GPU accelerated code for Penning trap simulations, which we extended to include the molecule-light interaction, we show that this scheme can decrease the time required for cooling by an order of magnitude with respect to Doppler cooling. In our simulation we found that a reduction of the axial anion temperature from $10 \mathrm{~K}$ to $50 \mathrm{mK}$ in around $10 \mathrm{~s}$ is possible. The temperature of the radial degrees of freedom was seen to thermalize to $150 \mathrm{mK}$. Based on the laser-cooled $\mathrm{C}_{2}{ }^{-}$, a study on the sympathetic cooling of anions with masses 1-50 nucleon was performed, covering relevant candidates for investigations of chemical anion reactions at ultracold temperatures as well as for antimatter studies.
\end{abstract}

DOI: 10.1103/PhysRevA.96.031401

Laser cooling of anions, which has so far never been achieved, would enable the sympathetic cooling of any other negatively charged species and open new opportunities in a variety of research areas. This includes research on the atmosphere and the interstellar medium [1], chemistry of highly correlated systems [2], and cooling of antiprotons for antimatter experiments [3]. Given the broad impact, several groups are exploring different approaches to cool anions [4-6].

Up to now, work on the cooling of anions has focused mainly on the atomic species $\mathrm{La}^{-}$and $\mathrm{Os}^{-}$[5-7] and the molecular species $\mathrm{C}_{2}^{-}$[4]. They all have in common that the dipole transitions attractive for the cooling can have narrow natural linewidths in the range of several kilohertz. Thus, employing traditional Doppler cooling would result in cooling times on the order of $100 \mathrm{~s}$ to reach the $100-\mathrm{mK}$ regime even when starting with particles precooled to the liquid helium temperature of $4 \mathrm{~K}$. This can be a problem when the cooling is in competition with heating effects stemming from, e.g., trap misalignment or collisions with residual gases.

Addressing this problem, we discuss the possibility of using the ac Stark shift for Sisyphus cooling in ion traps [8]. Although also applicable to positive ions, we will concentrate on the specific case of $\mathrm{C}_{2}{ }^{-}$in a Penning trap, which is an interesting candidate for sympathetic cooling of antiprotons $[3,4]$. We will describe the cooling scheme in detail below. Using this method, depending on the laser power available, the energy removed per scattered photon can be much higher than by using the Doppler effect. This results in an accelerated cooling, while also easing the requirements on repumping, which is of special importance for the cooling of molecules.

The level structure of $\mathrm{C}_{2}{ }^{-}$is well known and depicted in Fig. 1(a) [10-13]. Given its homonuclear character, only elec-

\footnotetext{
*julian.valentin.fesel@cern.ch
}

Published by the American Physical Society under the terms of the Creative Commons Attribution 4.0 International license. Further distribution of this work must maintain attribution to the author(s) and the published article's title, journal citation, and DOI. tronic transitions are allowed, resulting in two possible systems for cooling. The first consists of the $B^{2} \Sigma\left(v^{\prime}=0, N^{\prime}=0\right) \leftrightarrow$ $X^{2} \Sigma\left(v^{\prime \prime}=0, N^{\prime \prime}=2\right)$ transition at $541 \mathrm{~nm}$, with a branching ratio of $72 \%$ for spontaneous decay via this channel and a natural linewidth of $\Gamma=2 \pi \times 2.1 \mathrm{MHz}$. In order to create a closed cycle for the cooling it is then necessary to repump several lower vibrational states $X^{2} \Sigma\left(v^{\prime \prime}=0, \ldots, 4\right)$, which was simulated for the case of Doppler cooling in a Paul trap in [4]. In the case of a Penning trap high magnetic fields of several tesla result in an additional strong splitting of the rotational sublevels, making the experimental realization of the repumping challenging.

We will therefore focus on the second possible system for cooling. This consists of the $A^{2} \Pi\left(v^{\prime}=0\right) \leftrightarrow X^{2} \Sigma\left(v^{\prime \prime}=0\right)$ transition at $2.54 \mu \mathrm{m}$, with a branching ratio of $96 \%$ and a natural linewidth of $\Gamma=2 \pi \times 3.1 \mathrm{kHz}$. Doppler cooling on this transition was estimated to take $\sim 300 \mathrm{~s}$ to reach the millikelvin range when starting from $10 \mathrm{~K}$ [14]. Another interesting scheme was discussed in [4] for a low-density plasma, using a magnetic-field gradient in a Penning trap in order to implement a Zeeman-shift-based Sisyphus cooling cycle. Comparing this method with our proposed scheme, the magnetic version has the benefit of a higher energy being removed per photon, leading to fast cooling rates in the millisecond range and thereby a lower sensitivity to environmental heating. Despite that, the magnetic inhomogeneity can lead to particle trapping due to the magnetic mirror effect, which is more pronounced for high-density plasmas and high-magnetic-field gradients. This can lead to ions being removed from the cooling cycle and makes a careful analysis necessary in that regime $[15,16]$. Using a strong laser to shift the energy levels as proposed in this work has the benefit of being all optical and independent of the trapping dynamics. Besides avoiding the complexity of a magnetic mirror, it is also a solution for experimental circumstances where a magnetic-field gradient cannot be implemented.

An extensive theoretical discussion of the ac Stark shift and dipole forces can be found in $[17,18]$. The proposed cooling scheme is illustrated in Fig. 1(b) using the same parameters as in the actual simulation as described below. 

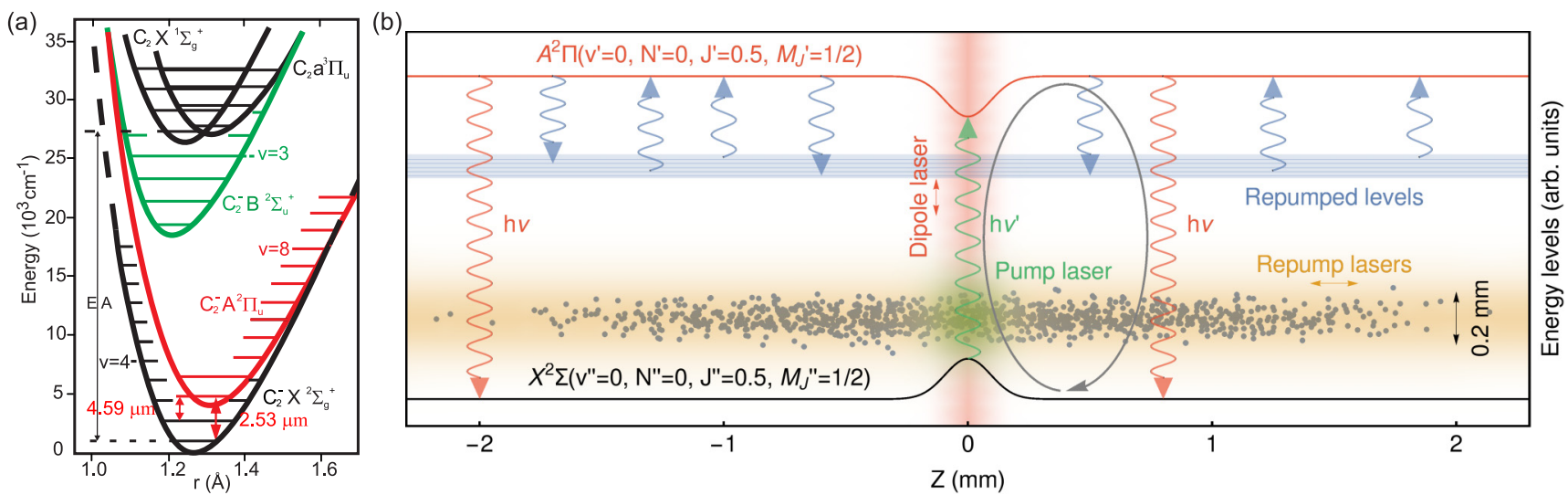

FIG. 1. (a) Overview of the electronic and vibrational states. The relevant transitions from the $A^{2} \Pi$ state for our cooling scheme are indicated. The electron affinity is equal to $3.27 \mathrm{eV}$ [9]. (b) Sketch of the Sisyphus cooling cycle to scale with simulation parameters. The geometry of the ion cloud (gray) is shown together with laser beam profiles (red, green, and yellow) and an overlay of the involved electronic levels (see also Fig. 2). A 6-W laser (red), detuned from the resonance of the $A^{2} \Pi\left(v^{\prime}=0, N^{\prime}=1, J^{\prime}=0.5, M_{J}^{\prime}=1 / 2\right) \leftrightarrow X^{2} \Sigma\left(v^{\prime \prime}=0, N^{\prime \prime}=0, J^{\prime \prime}=0.5, M_{J}^{\prime \prime}=1 / 2\right)$ transition by $\delta=1 \mathrm{GHz}$ and with a waist of $w_{\text {dip }}=185 \mu \mathrm{m}$, shifts the levels locally around $z=0$, thereby creating a potential $U_{\text {dip }}=12.4 \mathrm{mK}$. A second laser (green) on resonance with the maximally shifted levels causes transitions to the upper state in particles in the maximum of the dipole potential, by absorbing a photon with energy $h v^{\prime}$. Due to the long lifetime of $50 \mu$ s of the $A^{2} \Pi(v=0)$ state in relation to their thermal energy, the particles are likely to leave the region of the shift and then decay to the initial state by emitting a photon with energy $h v=h v^{\prime}+U_{\text {dip }}$. Repump lasers (orange) on all other levels prevent losses from the cooling cycle by particles decaying to dark states. The whole cycle effectively removes the energy $\Delta E=h\left(v-v^{\prime}\right)$ from the particles.

Aside from repumpers, it is based on two lasers that address the $A^{2} \Pi\left(v^{\prime}=0, N^{\prime}=1, J^{\prime}=0.5, M_{J}^{\prime}=1 / 2\right) \leftrightarrow X^{2} \Sigma\left(v^{\prime \prime}=\right.$ $\left.0, N^{\prime \prime}=0, J^{\prime \prime}=0.5, M_{J}^{\prime \prime}=1 / 2\right)$ transition of $\mathrm{C}_{2}{ }^{-}$. One is high powered in the range of several watts and blue detuned from resonance by $1 \mathrm{GHz}$ [which we will refer to as a dipole laser (DL)], thereby shifting the energetic levels. A second laser is tuned to be resonant with the shifted levels [referred to as a pumping laser (PL)] and overlapped with the DL and the molecules. The scheme could also be realized with a single broad and strong laser, used for shifting and pumping at the same time, but in this work we use two separate lasers to permit greater flexibility. If only a single laser is used for pumping and shifting, Doppler heating by the blue-detuned DL has to be taken into account.

Focusing the lasers to a narrow region along the axis of the trap then creates an area where particles are pumped to the excited state $A^{2} \Pi\left(v^{\prime}=0\right)$. If the lifetime of this state is long enough, a large fraction of particles stay in the excited state until they have left the region of the lasers and spontaneously decay to one of the lower states. In order to close the cooling cycle the lower levels, with the exception of $X^{2} \Sigma\left(v^{\prime \prime}=\right.$ $\left.0, N^{\prime \prime}=0, J^{\prime \prime}=0.5, M_{J}^{\prime \prime}=1 / 2\right)$, are continuously repumped to the excited state by lasers applied in the axial direction, eventually resulting in a spontaneous decay to this ground state via the emission of a photon at frequency $v$. The particles stay in this state until they reenter the shifting region. The whole cycle effectively removes the energy $\Delta E=h\left(v-v^{\prime}\right)$ from the particles. The energy difference stems from the axial kinetic energy of the particles and is removed when entering and leaving the shifting region due to the dipole force exerted by the laser.

Our simulation is based on the SIMBUCA code $[19,20]$, which was specifically developed for the simulation of particles in a Penning trap. It uses a GPU to parallelize the calculation of the Coulomb interaction, allowing one to increase the total number of particles in comparison with a CPU-based simulation. The trajectories of the particles in the trap, including the cyclotron motion, are fully resolved using a fifth-order Dormand-Prince integrator. In order to include the interaction of the molecules with the light fields, we extended the code to include a simulation of the laser-induced electronic population transfers, which we based on rate equations and the Monte Carlo method. This approach is well justified in the regime of lasers with a spectral distribution much broader than the natural linewidth $[21,22]$. Our code treats the populations $\Pi_{i}$ of all 16 levels (see Fig. 2, details described later in the text) separately according to the equations

$$
\begin{aligned}
\dot{\Pi}_{i} & =\Sigma_{j}\left[\gamma_{i j}(\mathbf{x})\left(\Pi_{j}-\Pi_{i}\right)+\Gamma_{j i} \Pi_{j}-\Gamma_{i j} \Pi_{i}\right], \\
\gamma_{i j}(\mathbf{x}) & =\frac{\Gamma_{i j} c^{2}}{16 \pi^{2} h \tau_{\mathrm{A}} v_{i j}^{3}} \rho_{i j}\left(v_{i j}\right) I_{i j}(\mathbf{x}),
\end{aligned}
$$

with $\rho_{i j}$ and $I_{i j}$ being the spectral intensity distribution of the lasers addressing the transition, $\Gamma_{i j}$ the respective Einstein coefficient, and $\tau_{\mathrm{A}}$ the lifetime of the excited state. The dipole force in the case of negligible scattering by the DL is given by

$$
\begin{aligned}
\mathbf{F}_{i}(\mathbf{x}) & =-\Sigma_{j} \frac{\hbar \delta_{i j}}{2} \nabla \ln \left(1+\frac{\omega_{i j}^{2}(\mathbf{x})}{2 \delta_{i j}^{2}}\right), \\
\omega_{i j}^{2}(\mathbf{x}) & =\frac{3 c^{2} \Gamma_{i j}}{2 \pi h v_{i j}^{3}} I_{i j}(\mathbf{x}),
\end{aligned}
$$

with $\omega_{i j}$ being the Rabi frequency.

For the simulation an ion cloud of $1024 \mathrm{C}_{2}{ }^{-}$molecules was initialized in thermal equilibrium at $T_{\text {init }}=10 \mathrm{~K}$, with a density of $n_{\text {init }}=2 \times 10^{13} \mathrm{~m}^{-3}$ and a confining magnetic field of $B=5 \mathrm{~T}$ in the Penning trap. The dimensions of the ion cloud are visualized in Fig. 3 with a mean radius of $\langle r\rangle=50 \mu \mathrm{m}$ and mean absolute axial extent of $\langle|z|\rangle=$ 


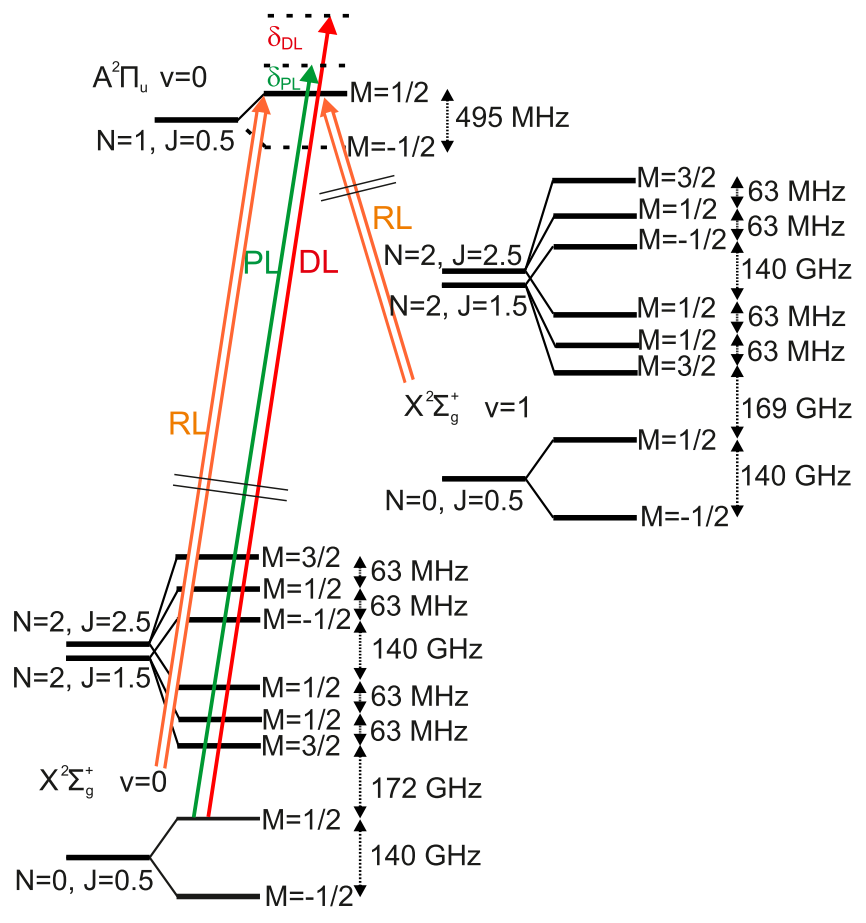

FIG. 2. Detail of the $A^{2} \Pi\left(v^{\prime}=0\right) \leftrightarrow X^{2} \Sigma\left(v^{\prime \prime}=0\right)$ transition and the intermediate $X^{2} \Sigma(v=1)$ states in a 5-T magnetic field. Several arrows indicate the transitions that are addressed by the different lasers described in the text.

$0.7 \mathrm{~mm}$. These parameters can be experimentally achieved by supersonic expansion with subsequent dielectric barrier discharge of acetylene gas in a neon carrier [12,23,24]. We expect this to produce internally cold $\mathrm{C}_{2}{ }^{-}$with evenly distributed kinetic energies in the sub-eV range [25]. After mass selection in the $10-\mathrm{eV}$ range the anions can be trapped and sympathetically cooled by electrons. Assuming a cryogenic 5-T Penning trap at $10 \mathrm{~K}$, temperatures around $100 \mathrm{~K}$ can be achieved in a few seconds [26]. Subsequent evaporative cooling allows for a preparation of the anions at $10 \mathrm{~K}$ [27].

The laser parameters were chosen in order to correspond to values that could be realistically achieved with existing technology. For the DL we used a power of $P_{\mathrm{DL}}=3 \mathrm{~W}$ along the radial $X$ axis, focused to a waist of $w_{\text {dip }}=185 \mu \mathrm{m}$ in the center of the ion cloud. In our simulation we compared two different settings for the DL, once as a free Gaussian beam and once modulated by a cosine squared of the right periodicity, so as to correspond to, e.g., the light in an enhancement cavity. The detuning from resonance was set to $\delta_{\mathrm{DL}}=1 \mathrm{GHz}$. In order for Eq. (2) to be valid, scattering by the DL needs to be negligible, which implies that the spectral width of the DL must be much smaller than $\delta_{\mathrm{DL}}$. For the chosen parameters and molecules at $50 \mathrm{mK}$, we estimated the scattering by the DL to be less than a single photon per pass through the laser. Since the DL is blue detuned, each scattered photon results in a Doppler heating of $62 \mathrm{nK}$. Given that these scattering events still contribute to the dipole cooling cycle and a dipole force potential of $U_{\text {dip }}=6.6 \mathrm{mK}$, we neglected this effect in the simulation.
The PL was modeled as two counterpropagating free beams along the radial $Y$ axis with $P_{\mathrm{PL}}=4 \mathrm{~mW}$, focused to the same size as the DL in the center of the ion cloud while neglecting the standing-wave pattern (SWP). The spectral width was set to $\Delta_{\mathrm{PL}}=120 \mathrm{MHz}$ FWHM with a detuning that corresponds to the level shift in the focus of the DL of $\delta_{\mathrm{PL}}=137 \mathrm{MHz}$. On resonance, a pumping rate of $\gamma=$ $10 \mathrm{MHz}$ is reached in the focus of the laser. The repumping lasers along the trap axis were set to be on resonance with a power of $2 \mathrm{~mW}$, the same waist as the other two lasers and a spectral width of $\Delta_{\mathrm{P}}=50 \mathrm{MHz}$. Depending on the transitions, the resulting pumping rate varies between $\gamma=$ $200 \mathrm{kHz}$ and $\gamma=2 \mathrm{MHz}$ in the focus of the lasers. The widths of the PL and repumpers need to be large enough in order to sufficiently address the ion cloud, taking into account Doppler broadening and the spacial spectral shift in the center of the DL. Closing the cooling cycle requires repumping of 15 levels (see Fig. 2), which is implemented in the simulation with separate light fields. Three are needed to cover the unused $X^{2} \Sigma\left(v^{\prime \prime}=(0,1), N^{\prime \prime}=0, J^{\prime \prime}=0.5, M_{J}^{\prime \prime}= \pm 1 / 2\right)$ levels and the remaining ones distributed onto the $X^{2} \Sigma\left[v^{\prime \prime}=\right.$ $\left.(0,1), N^{\prime \prime}=2, J^{\prime \prime}=(1.5,2.5), M_{J}^{\prime \prime}=( \pm 1 / 2, \pm 3 / 2)\right]$ states. In an experimental realization this could be done using three lasers. A single one is scanned over the $X^{2} \Sigma\left(v^{\prime \prime}=1\right)$ states, which is sufficient due to the low branching ratio of $4 \%$. By imprinting sidebands at $63 \mathrm{MHz}$ a second laser can repump the $X^{2} \Sigma\left(v^{\prime \prime}=0, N^{\prime \prime}=2\right)$ levels by scanning between the two branches of $X^{2} \Sigma\left[v^{\prime \prime}=(0,1), N^{\prime \prime}=2, J^{\prime \prime}=(1.5,2.5)\right]$, leaving an additional laser for the strong transition from $X^{2} \Sigma\left(v^{\prime \prime}=0, N^{\prime \prime}=0, J^{\prime \prime}=0.5, M_{J}^{\prime \prime}=-1 / 2\right)$.

The interesting cooling dynamics happen on a time scale of $14 \mathrm{~s}$, which leads to impractically long simulation times. In order to circumvent this problem, we introduce an artificial augmentation factor $f_{\text {sc }}$ to scale the size of the dipole force acting on the particles, which is initially set to 10 and consecutively reduced to unity at $\left[t(\mathrm{~ms}), f_{\mathrm{sc}}\right]=[(0,10),(600,2),(925,1)]$ for the case without and at $\left[t(\mathrm{~ms}), f_{\mathrm{sc}}\right]=[(0,10),(800,2),(2750,1)]$ for the case including the SWP. This effectively increases the dipole force potential, while leaving the pumping rates and the detuning the same. The reduction is necessary, since an artificially large force will start to prevent particles from reaching the intensity maximum of the DL, thereby removing them from the cooling, when its potential amplitude becomes comparable to the particle thermal energy. During our simulation the ratio of the thermal energy to the scaled dipole force potential was always larger than 10 . Since the remaining small influence on the cooling speed is detrimental, the simulation still gives an upper bound on the efficiency. The actual computation simulates the trap dynamics during $8.8 \mathrm{~s}$ without and $5.5 \mathrm{~s}$ with a SWP.

Figure 3 shows the simulated temperature evolution. The time axis was scaled by the amplification factor $f_{\mathrm{sc}}$ to show the cooling time under realistic conditions. The resulting smooth behavior of the temperature is a powerful indication that the scaling factor is well justified and does not introduce any significant nonlinear effects. Two solid lines indicate the initial linear cooling behavior and the deviation from it as the ions get colder. For the axial degree of freedom this can be explained with the decrease in velocity in relation to the lifetime $\tau_{\mathrm{A}}$ of the 

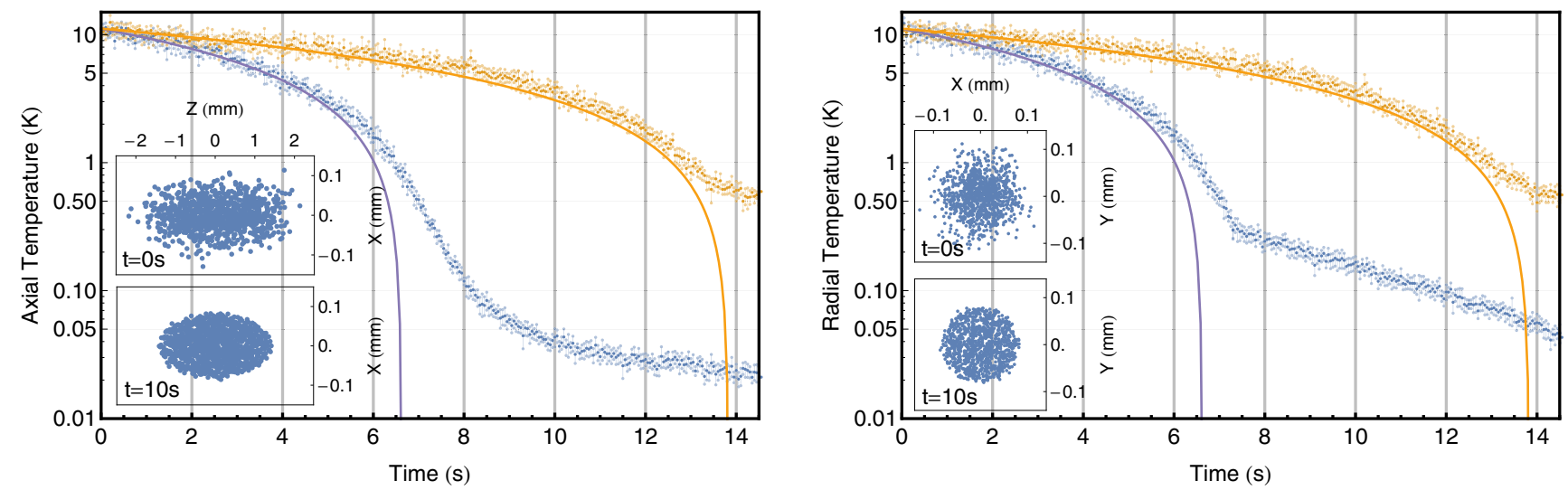

FIG. 3. Plasma temperature plot of $1024 \mathrm{C}_{2}{ }^{-}$molecules subjected to dipole force cooling, once without (blue lower symbols) and once including a SWP (yellow upper symbols). The temperature is derived by fitting a Boltzmann distribution to the velocity histogram of the axial and radial degrees of freedom in cylindrical coordinates. The two solid lines show linear fits to the initial temperature evolution in order to illustrate the deviation from linear behavior as the molecules get colder. The insets show projected snapshots of the plasma taken from the simulation without a SWP at two points in time.

upper state. This gradually reduces the probability for a decay outside the shifted region and thereby the efficiency of the cooling. At a temperature of $T_{w} \simeq 60 \mathrm{mK}$ the mean traveled distance during the time $\tau_{\mathrm{A}}$ becomes comparable to the waist of the DL. Together with the depth of the dipole force potential, this poses a practical limit to the cooling without readjustment of the laser parameters. The different cooling performance for the two cases with and without the SWP can be attributed to two factors. The first is a simple geometric advantage of the case without the SWP, since the overlap of the resonant region with the anion cloud is smaller. The second factor is an additional heating source that is caused by the SWP. Applying the dipole force potential in the Penning trap effectively causes a small inhomogeneity in the radially symmetric potential of the Penning trap. This causes a small expansion of the ion cloud and radial heating, which is more pronounced in the case with the SWP and stops the cooling at around $500 \mathrm{mK}$. For the case without the SWP the nonlinear effects in the radial temperature and the deviation from the evolution of the axial pendant can be attributed to a reduced thermalization between the axial and radial degrees of freedom starting in the weakly coupled regime at lower temperatures. The plasma in our simulation is initialized in the weak-coupling regime at $\Gamma_{\mathrm{c}}=\frac{1}{4 \pi \epsilon_{0}} \frac{e^{2}}{k T}\left(\frac{4 \pi n}{3}\right)^{1 / 3} \ll 1$ [28]. At around $T \simeq 0.5 \mathrm{~K}$ it reaches the weakly coupled regime $\Gamma_{\mathrm{c}} \simeq 1$ and is strongly coupled $\left(\Gamma_{\mathrm{c}} \gg 1\right)$ towards the end of the simulation. As discussed in $[29,30]$, the equipartition decreases exponentially in the weakly coupled regime for a strongly magnetized plasma $\frac{\Omega_{\mathrm{c}} b}{\bar{v}} \gg 1$. Apart from exhibiting interesting plasma dynamics, this shows that there is no significant direct cooling of the radial degrees of freedom.

An important concern for a cooling scheme working on a time scale of $14 \mathrm{~s}$ is heating by the environment. For a room temperature setup and at a pressure of around $\sim 10^{-9} \mathrm{~Pa}$, this was experimentally investigated in [30,31], where it was concluded that the dominant contribution to heating stems from collisions of the trapped ions with residual gases. A heating rate of around $\sim 0.1 \mathrm{~K} \mathrm{~s}^{-1}$ was measured, which would have a significant influence on the cooling speed and the final temperature of the described scheme. By going to a cryogenic Penning trap in the region of $10 \mathrm{~K}$, where pressure down to $\sim 10^{-14} \mathrm{~Pa}[32]$ is achieved, the heating rate can be much lower with a reported value of $5 \times 10^{-6} \mathrm{~K} \mathrm{~s}^{-1}$ [33]. In the presented simulation we assumed a cryogenic environment and neglected heating effects.

An interesting application of laser-cooled anions would be as a sympathetic coolant for other negatively charged species. Since Penning traps can be used to store species with widely differing masses together, they are well suited for this task. We investigated the efficiency of sympathetic cooling for a broad mass range with $\mathrm{C}_{2}{ }^{-}$as a coolant. For this we initialized the same ion cloud as for the previous laser cooling simulation, with $20 \%$ of the particles being replaced by anions of a different mass $m^{\prime}$ that are not directly addressed by the cooling. In order to speed up the simulation, the original full treatment of the laser cooling is approximated by an artificial drag force that resembles the actual cooling dynamics and is given by $F_{\mathrm{z}}=-\operatorname{sgn}\left(v_{\mathrm{z}}\right) U_{0} \exp \left(-2 z^{2} / w^{2}\right)$. The parameters are chosen so as to allow a simulation of particles with a mass corresponding to antiprotons. Figure 4 shows the results of the sympathetic cooling of different masses $m^{\prime}$ ranging from 1 to 50 nucleon. Even for an enhanced cooling during $100 \mathrm{~ms}$, the sympathetic cooling of the axial degree of freedom is sufficiently fast. As for the previous simulation, a reduction in the equipartition of the radial degrees can be seen at lower temperatures. On the time scale of an actual implementation of the dipole cooling scheme, which is two orders of magnitude larger, the equipartition can be expected to reach lower temperatures.

In summary, we have presented an all-optical cooling scheme that is suitable for a variety of ion traps. Our simulation of the specific case of $\mathrm{C}_{2}{ }^{-}$in a Penning trap, which is a promising candidate for sympathetic cooling of other anions, shows a reduction in cooling time by an order of magnitude in comparison with Doppler cooling. On a time scale of $10 \mathrm{~s}$ the anions reach a temperature of $50 \mathrm{mK}$ in the directly cooled axial and $150 \mathrm{mK}$ in the indirectly cooled radial degrees of freedom. Similar improvements should be achievable for 

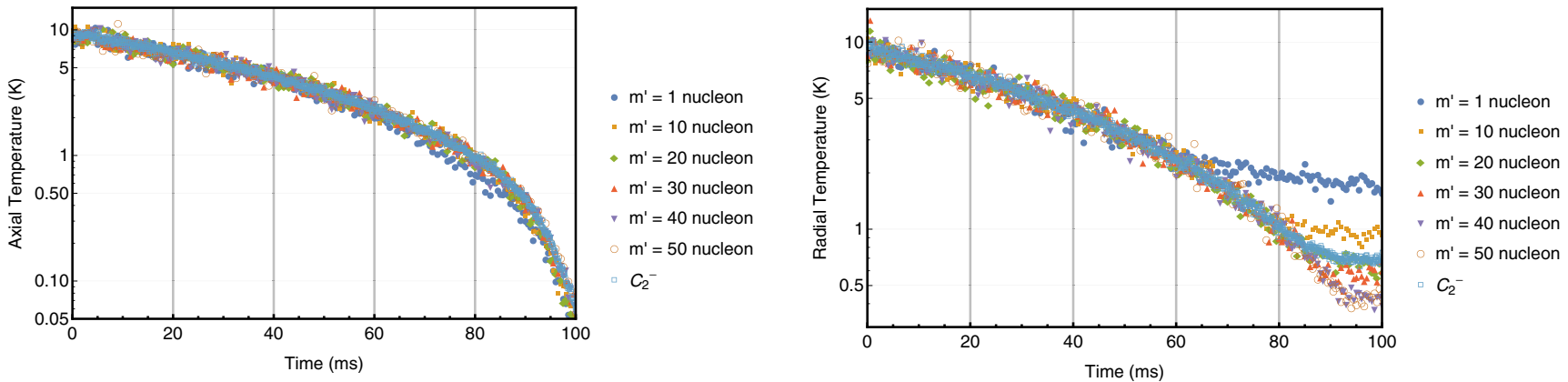

FIG. 4. Results of the sympathetic cooling of 200 negatively charged species with a mass $m^{\prime}$ immersed in an ion cloud of $824 \mathrm{C}_{2}{ }^{-}$. For reference, the temperature of the $\mathrm{C}_{2}{ }^{-}$is given from the simulation involving the largest mass with $m^{\prime}=50$ nucleon. The cooling of the $\mathrm{C}_{2}{ }^{-}$ was implemented using an artificial drag force that resembles a dipole cooling scheme.

other ions exhibiting narrow cooling transitions. Furthermore, we investigated the efficiency of sympathetic cooling by laser-cooled $\mathrm{C}_{2}{ }^{-}$. A mass range of 1-50 nucleon was covered, which includes light anions like antiprotons and heavier anions relevant in ultracold chemistry. Another promising application of the described scheme could be in the production of ultracold electrons. A transition to a cryocooled Penning trap at $T=1 \mathrm{~K}$ and with a magnetic field of $B=0.5 \mathrm{~T}$ would reduce heating by synchrotron radiation [34] below the axial cooling rate found in this work. Together with an increased equipartition rate at lower magnetic fields [30], this could potentially allow one to decrease the particle mass range where this cooling scheme is applicable down to the regime of electrons.

We want to thank our summer student Cui Hao for his help with writing the code. The research leading to these results received funding from the European Research Council under Grant Agreement No. 277762 COLDNANO.
[1] T. P. Snow and V. M. Bierbaum, Annu. Rev. Anal. Chem. 1, 229 (2008).

[2] O. Dulieu and C. Gabbanini, Rep. Prog. Phys. 72, 086401 (2009).

[3] A. Kellerbauer and J. Walz, New J. Phys. 8, 45 (2006).

[4] P. Yzombard, M. Hamamda, S. Gerber, M. Doser, and D. Comparat, Phys. Rev. Lett. 114, 213001 (2015).

[5] A. Kellerbauer, A. Fischer, and U. Warring, Phys. Rev. A 89, 043430 (2014).

[6] E. Jordan, G. Cerchiari, S. Fritzsche, and A. Kellerbauer, Phys. Rev. Lett. 115, 113001 (2015).

[7] L. Pan and D. R. Beck, Phys. Rev. A 82, 014501 (2010).

[8] V. V. Ivanov and S. Gupta, Phys. Rev. A 84, 063417 (2011).

[9] M. K. Ervin and W. C. Lineberger, J. Phys. Chem. 95, 1167 (1991).

[10] P. Rosmus and H.-J. Werner, J. Chem. Phys. 80, 5085 (1984).

[11] T. Sedivcová and V. Spirko, Mol. Phys. 104, 1999 (2006).

[12] P. L. Jones, R. D. Mead, B. E. Kohler, S. D. Rosner, and W. C. Lineberger, J. Chem. Phys. 73, 4419 (1980).

[13] Y. Shan-Shan, Chin. Phys. 12, 745 (2003).

[14] S. Gerber, J. Fesel, M. Doser, D. Comparat (unpublished).

[15] J. Fajans, Phys. Plasmas 10, 1209 (2003).

[16] K. Gomberoff, J. Fajans, J. Wurtele, A. Friedman, D. P. Grote, R. H. Cohen, and J. L. Vay, Phys. Plasmas 14, 052107 (2007).

[17] J. Dalibard and C. Cohen-Tannoudji, J. Opt. Soc. Am. B 2, 1707 (1985).

[18] R. Grimm, M. Weidemüller, and Y. Ovchinnikov, Adv. At. Mol. Opt. Phys. 42, 95 (2000).

[19] S. Van Gorp, M. Beck, M. Breitenfeldt, V. De Leebeeck, P. Friedag, A. Herlert, T. Iitaka, J. Mader, V. Kozlov, S. Roccia,
G. Soti, M. Tandecki, E. Traykov, F. Wauters, C. Weinheimer, D. Zákoucký, and N. Severijns, Nucl. Instrum. Methods Phys. Res. Sect. A 638, 192 (2011).

[20] S. Van Gorp and P. Dupre, in Non-Neutral Plasma Physics VIII: 10th International Workshop on Non-Neutral Plasmas, edited by X. Sarasola, L. Schweikhard, and T. S. Pedersen, AIP Conf. Proc. No. 1521 (AIP, New York, 2013), pp. 300-308.

[21] R. Höppner, E. Roldán, and G. J. de Valcárcel, Am. J. Phys. 80, 882 (2012).

[22] D. Comparat, Phys. Rev. A 89, 043410 (2014).

[23] U. Even, EPJ Tech. Instrum. 2, 17 (2015).

[24] H. Hotop, T. A. Patterson, and W. C. Lineberger, Phys. Rev. A 8, 762 (1973).

[25] U. Even (private communication).

[26] S. L. Rolston and G. Gabrielse, Hyperfine Interact. 44, 233 (1988).

[27] G. B. Andresen et al., Phys. Rev. Lett. 105, 013003 (2010).

[28] D. H. E. Dubin and T. M. O’Neil, Rev. Mod. Phys. 71, 87 (1999).

[29] D. H. E. Dubin, Phys. Rev. Lett. 94, 025002 (2005).

[30] M. J. Jensen, T. Hasegawa, J. J. Bollinger, and D. H. E. Dubin, Phys. Rev. Lett. 94, 025001 (2005).

[31] M. J. Jensen, T. Hasegawa, and J. J. Bollinger, Phys. Rev. A 70, 033401 (2004).

[32] A. Mooser, S. Ulmer, K. Blaum, K. Franke, H. Kracke, C. Leiteritz, W. Quint, C. C. Rodegheri, C. Smorra, and J. Walz, Nature (London) 509, 596 (2014).

[33] G. B. Andresen et al., Nat. Phys. 7, 558 (2011).

[34] J. Bernard, J. Alonso, T. Beier, M. Block, S. Djekić, H.-J. Kluge, C. Kozhuharov, W. Quint, S. Stahl, T. Valenzuela, J. Verdú, M. Vogel, and G. Werth, Nucl. Instrum. Methods Phys. Res. Sect. A 532, 224 (2004). 